\title{
BMJ Open Rationale and study design of the Prospective comparison of Angiotensin Receptor neprilysin inhibitor with Angiotensin receptor blocker MEasuring arterial sTiffness in the eldERly (PARAMETER) study
}

\author{
Bryan Williams, ${ }^{1}$ John R Cockcroft, ${ }^{2}$ Kazuomi Kario, ${ }^{3}$ Dion H Zappe, ${ }^{4}$ \\ Pamela Cardenas, ${ }^{4}$ Allen Hester, ${ }^{4}$ Patrick Brunel, ${ }^{5}$ Jack Zhang ${ }^{4}$
}

To cite: Williams B,

Cockcroft JR, Kario K, et al. Rationale and study design of the Prospective comparison of Angiotensin Receptor neprilysin inhibitor with Angiotensin receptor blocker MEasuring arterial sTiffness in the eldERly (PARAMETER) study. BMJ Open 2014;4: e004254. doi:10.1136/ bmjopen-2013-004254

- Prepublication history and additional material for this paper is available online. To view these files please visit the journal online (http://dx.doi.org/10.1136/ bmjopen-2013-004254)

Received 15 October 2013 Revised 8 December 2013 Accepted 11 December 2013

CrossMark

For numbered affiliations see end of article.

Correspondence to Professor Bryan Williams; bryan.williams@ucl.ac.uk

\section{ABSTRACT}

Introduction: Hypertension in elderly people is characterised by elevated systolic blood pressure (SBP) and increased pulse pressure (PP), which indicate large artery ageing and stiffness. LCZ696, a first-in-class angiotensin receptor neprilysin inhibitor (ARNI), is being developed to treat hypertension and heart failure. The Prospective comparison of Angiotensin Receptor neprilysin inhibitor with Angiotensin receptor blocker MEasuring arterial sTiffness in the eldERly

(PARAMETER) study will assess the efficacy of LCZ696 versus olmesartan on aortic stiffness and central aortic haemodynamics.

Methods and analysis: In this 52-week multicentre study, patients with hypertension aged $\geq 60$ years with a mean sitting (ms) SBP $\geq 150$ to $<180$ and a

$\mathrm{PP}>60 \mathrm{~mm} \mathrm{Hg}$ will be randomised to once daily LCZ696 $200 \mathrm{mg}$ or olmesartan $20 \mathrm{mg}$ for 4 weeks, followed by a forced-titration to double the initial doses for the next 8 weeks. At 12-24 weeks, if the BP target has not been attained (msSBP $<140$ and $m s$ diastolic BP

$<90 \mathrm{~mm} \mathrm{Hg}$ ), amlodipine (2.5-5 mg) and subsequently hydrochlorothiazide $(6.25-25 \mathrm{mg})$ can be added. The primary and secondary endpoints are changes from baseline in central aortic systolic pressure (CASP) and central aortic PP (CAPP) at week 12, respectively. Other secondary endpoints are the changes in CASP and CAPP at week 52. A sample size of 432 randomised patients is estimated to ensure a power of $90 \%$ to assess the superiority of LCZ696 over olmesartan at week 12 in the change from baseline of mean CASP, assuming an SD of $19 \mathrm{~mm} \mathrm{Hg}$, the difference of $6.5 \mathrm{~mm} \mathrm{Hg}$ and a $15 \%$ dropout rate. The primary variable will be analysed using a two-way analysis of covariance.

Ethics and dissemination: The study was initiated in December 2012 and final results are expected in 2015. The results of this study will impact the design of future phase III studies assessing cardiovascular protection.

Clinical trials identifier: EUDract number 2012-002899-14 and ClinicalTrials.gov NCT01692301.

\section{Strengths and limitations of this study}

- This is a randomised controlled trial of a new class of drug therapy (angiotensin receptor neprilysin inhibitor) for hypertension versus a comparator that blocks only the angiotensin receptor-this will inform on the added value of neprilysin inhibition in the context of systolic hypertension.

- The study incorporates a detailed clinical experimental medicine mechanistic study that will interrogate the actions of this new drug class on vascular haemodynamics and function.

- The study evaluates a novel treatment approach for a major unmet clinical need, that is, systolic hypertension.

- The study has inadequate statistical power to assess the impact of the interventions on major clinical outcomes beyond blood pressure and vascular haemodynamics and function.

\section{INTRODUCTION}

Hypertension accounts for 9.4 million cardiovascular $(\mathrm{CV})$ deaths annually worldwide and is affecting more than two-thirds of people aged $\geq 65$ years, an age group that is growing globally. ${ }^{12}$ The treatment of hypertension has been shown to reduce the risk of morbidity and mortality associated with elevated blood pressure (BP), including stroke, ischaemic heart disease, heart failure, chronic kidney disease and possibly cognitive decline. ${ }^{3}$ Despite the availability of multiple drug classes with different mechanisms of action, hypertension, especially systolic blood pressure (SBP), remains inadequately controlled. ${ }^{4-6}$

The SBP usually increases from childhood throughout life, while diastolic BP (DBP) 
remains relatively constant or decreases beyond 50-60 years of age. The changing patterns of $\mathrm{BP}$ throughout life reflect different pathologies. In the young, hypertension is predominantly due to an increased DBP and mean arterial pressure (MAP), as a result of a relative increase in cardiac output and/or increased peripheral vascular resistance. ${ }^{7}$ On the other hand, advancing age, beyond mid-life, is associated with an increased stiffness of large elastic arteries, especially the aorta. Arterial stiffening adversely affects the characteristic impedance of the aorta, requiring more cardiac work and raising SBP as more stroke volume is delivered during systole owing to the increased pulse wave velocity (PWV). DBP also decreases due to less elastic recoil leading to reduced flow, thus increasing pulse pressure (PP) independent of any changes in MAP. PWV been shown to be an independent predictor of CV outcomes, including mortality, ${ }^{8}$ myocardial infarction (MI) ${ }^{8}$ stroke, ${ }^{8}$ atrial fibrillation, ${ }^{9}$ cognitive decline ${ }^{10}$ and renal dysfunction, ${ }^{11}$ and more specifically aortic PWV (aPWV), a robust measure of aortic arterial stiffness, has been shown to predict the adverse CV outcomes. ${ }^{7}$

Another consequence of arterial ageing and stiffening is that the amplification of SBP and PP from the aortic root to the peripheral arteries diminishes. In a healthy arterial system, central aortic systolic pressure (CASP) and PP are amplified as they move towards the periphery, such that the measured brachial systolic pressure is typically around $10 \mathrm{~mm} \mathrm{Hg}$ higher than the corresponding aortic root pressure. ${ }^{12}$ With ageing, this amplification is reduced because of the increased PWV and the increase in the early wave reflection resulting in the measured brachial SBP and PP becoming closer to the corresponding aortic root pressures. Some studies have suggested that central pressures may have a closer correlation than peripheral BP with end-organ damage ${ }^{13-15}$ and $\mathrm{CV}$ risk, ${ }^{15}$ such as extent of coronary atherosclerosis, carotid intima-media thickness, left ventricular (LV) hypertrophy and LV diastolic function. ${ }^{14} 1617$

These observations raise the intriguing question as to whether treatments used to lower BP could differentially affect aortic pressure relative to brachial pressures and also arterial stiffness per se. It has been demonstrated that BP-lowering drugs can have marked differential effects on central aortic pressure (CAP) and brachial BP. $^{18}$ These effects mimic a functional antiageing effect in terms of their impact on wave-form morphology, and greater reduction in central pressures relative to brachial pressures. Intriguingly, the $\beta$-blockers, a drug class which was least effective at lowering aortic pressure also appeared to be the least effective class at reducing the risk of stroke in elderly patients. ${ }^{18}$ This supports the concept that the more effective lowering of aortic relative to brachial pressure may be clinically important.

Despite the findings cited above, controlling SBP remains the most important unmet need in the clinical management of hypertension. The rise in SBP and PP with ageing appears to be strongly related to arterial stiffening and increased impedance to flow through a stiff aorta. This suggests that the treatments targeting aortic stiffening and reducing characteristic impedance would be effective particularly at reducing systolic pressure. Early proof of this concept came from the studies with omapatrilat, a vasopeptidase inhibitor that simultaneously inhibits neprilysin and ACE. Neprilysin inhibition enhances natriuretic peptide (NP) levels by blocking their degradation. NP has vasodilating actions, which could reduce aortic stiffness, improve characteristic impedance and thereby reduce SBP and PP. Studies with omapatrilat show greater improvements in aortic characteristic impedance compared with enalapril, beyond the effects of BP lowering after 12 weeks of therapy. ${ }^{19}$ This benefit on aortic function was also associated with impressive data on SBP and PP lowering in patients with hypertension.

Although omapatrilat was withdrawn due to safety concerns, a proof of concept was established for concomitant inhibition of neprilysin and renin-angiotensin-aldosterone system (RAAS) with the potential to be an attractive treatment strategy to improve aortic haemodynamics. Increased NP levels also promote natriuresis and reduce sympathetic tone, together with antiproliferative and antihypertrophic effects and inhibition of aldosterone secretion. ${ }^{20}$ Alongside, suppression of RAAS would be complementary to neprilysin inhibition, which attenuates vasoconstriction, reduces sodium and water retention and also inhibits the development of $\mathrm{CV}$ hypertrophy and adverse remodelling.

Recently, LCZ696, a first-in-class angiotensin receptor neprilysin inhibitor (ARNI), has been developed. LCZ696 delivers systemic exposure to a neprilysin inhibitor prodrug, AHU377 (which rapidly converts into active LBQ657), and an angiotensin receptor blocker (ARB), valsartan. LCZ696 at 100, 200 and $400 \mathrm{mg}$ once daily, in patients with mild-to-moderate essential hypertension, resulted in greater $\mathrm{BP}$ reductions than corresponding doses of valsartan alone (160 and $320 \mathrm{mg}$ ) and was well tolerated. ${ }^{21}$ LCZ696 compared with valsartan was effective especially at reducing brachial SBP and PP. Furthermore, in patients with heart failure with preserved ejection fraction, LCZ696 has shown to reduce N-terminal-pro B-type natriuretic peptide (NT-proBNP), a biomarker of LV wall stress, to a greater extent than valsartan alone at 12 weeks and was well tolerated. ${ }^{22}$

Thus, the big challenge in hypertension treatment is to reduce the SBP, and the available evidence suggests that this could be achieved by improving the haemodynamic performance of the ageing aorta. The Prospective comparison of Angiotensin Receptor neprilysin inhibitor with Angiotensin receptor blocker MEasuring arterial sTiffness in the eldERly (PARAMETER) study is designed to compare the effect of LCZ696 with olmesartan, an ARB, on CASP, other measures of central aortic haemodynamics and arterial stiffness and ambulatory BPs in elderly patients with an elevated brachial SBP and a widened PP. The widened PP was chosen as an entry 
criteria as being indicative of aortic stiffening and advanced aortic ageing. The objective was to determine whether the ARNI LCZ696 can reverse some of the effects of arterial ageing in elderly patients with systolic hypertension, and thereby improve aortic pressures and haemodynamics. The study was initiated in December 2012 and the final results are expected in 2015. This manuscript describes the design, objectives and prespecified analysis plan for the PARAMETER study.

\section{METHODS}

Study design

The PARAMETER study is a 52-week, multicentre, randomised, double-blind, active-controlled, parallel-group study, involving 51 centres from 13 countries (Europe 47\%, South America 14\%, Asia 19\% and the USA 20\% - see online supplement for list of local investigators and participating centres). The study includes a screening period, a placebo run-in and an initial double-blind treatment period of 12 weeks with LCZ696 monotherapy, followed by a double-blind extension of 40 weeks, during which add-on therapy is allowed to reach the BP treatment goal. Patients will be randomised to receive either once daily LCZ696 $200 \mathrm{mg}$ or olmesartan $20 \mathrm{mg}$ for 4 weeks, followed by a forced titration to double the initial doses for the next 8 weeks. After 12 weeks, patients with uncontrolled BP (mean sitting (ms) SBP $>140$ and/or msDBP $>90 \mathrm{~mm} \mathrm{Hg}$ ) will be prescribed amlodipine (2.5-5 mg) and then hydrochlorothiazide $(6.25-25 \mathrm{mg})$ as needed, at an interval of 4 weeks up to week 24 (figure 1). This study has been approved by all relevant ethics committees.

\section{Study participants}

Elderly patients (aged $\geq 60$ years) with essential hypertension (either untreated or treated with antihypertensive agents) and patients who have msSBP $\geq 150$ and $<180 \mathrm{~mm} \mathrm{Hg}$ at randomisation are eligible for inclusion in the study. Untreated patients (if they are newly diagnosed or have not been treated with antihypertensive drugs for 4 weeks prior to screening) must have msSBP $\geq 150$ and $<180 \mathrm{~mm} \mathrm{Hg}$ at screening and randomisation, whereas patients who have been treated with antihypertensive agents 4 weeks prior to screening must have $\mathrm{msSBP} \geq 140$ and $<180 \mathrm{~mm} \mathrm{Hg}$ after 1 or 2 weeks of washout/placebo run-in and $\geq 150$ and $<180 \mathrm{~mm} \mathrm{Hg}$ at randomisation. All patients must have a $\mathrm{PP}>60 \mathrm{~mm} \mathrm{Hg}$ at randomisation. Patients with malignant or severe hypertension, secondary causes of hypertension, history of atrial fibrillation or atrial flutter during the 3 months prior to screening or active atrial fibrillation or atrial flutter on ECG, history of CV disease (eg, MI) during 12 months prior to screening and evidence of severe renal impairment (eg, estimated glomerular filtration rate $<30 \mathrm{~mL} / \mathrm{min} / 1.73 \mathrm{~m}^{2}$ ) are excluded-boxes 1 and 2 summarise the inclusion and exclusion criteria, respectively. Patients have to provide a written informed consent before starting any study-related procedures.

\section{The study objectives and endpoints}

The primary objective of the study is to demonstrate the superiority of an LCZ696-based treatment regimen over an olmesartan-based treatment regimen in reducing mean CASP after 12 weeks of treatment. Superiority testing is also planned for the key secondary efficacy

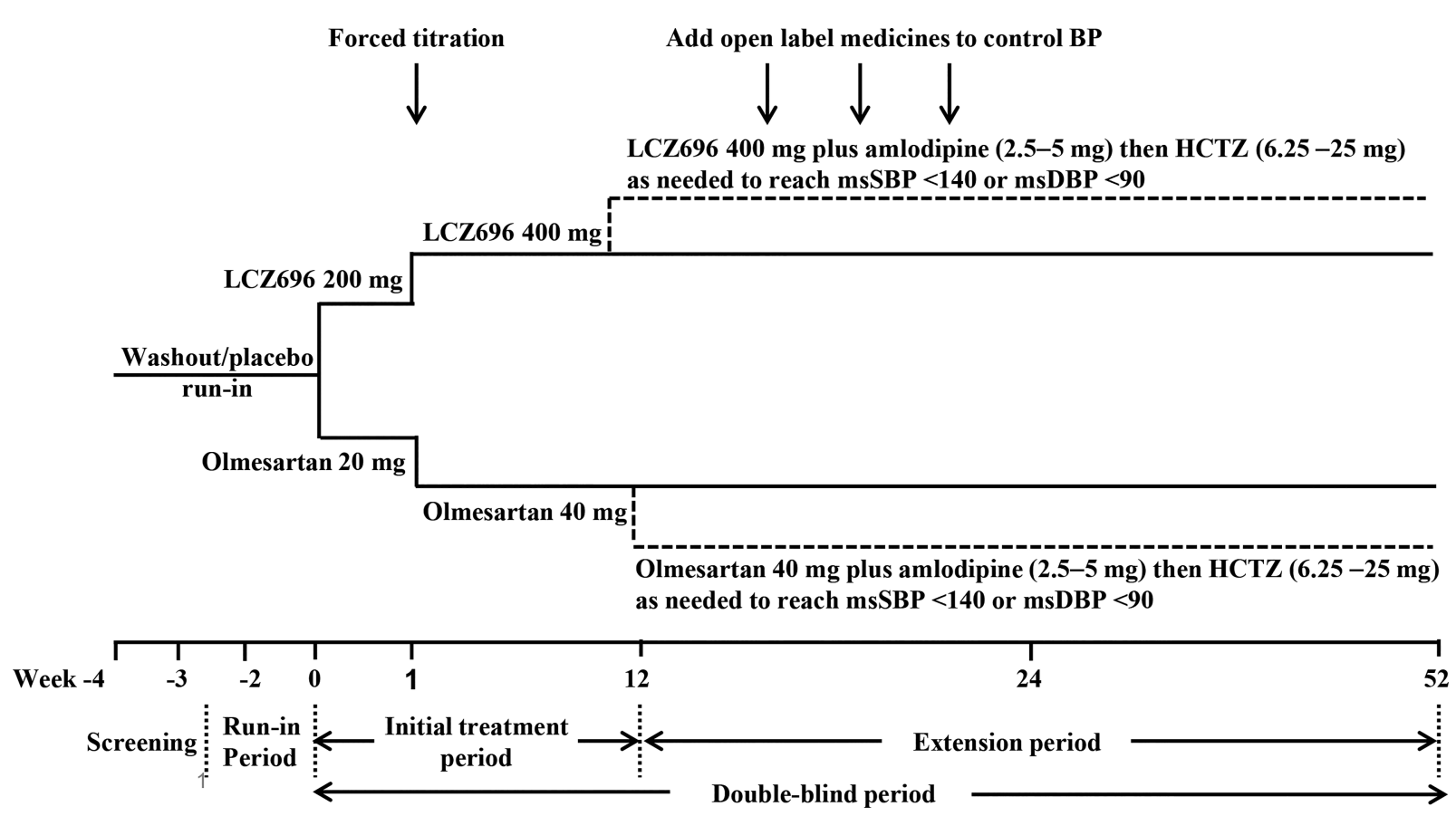

Figure 1 Study design. HCTZ, hydrochlorothiazide; msDBP, mean sitting diastolic blood pressure; msSBP, mean sitting systolic blood pressure. 
assessment, that is, the reduction in mean central aortic PP (CAPP) after 12 weeks of treatment, and other secondary efficacy assessments such as mean CASP and CAPP after 52 weeks of treatment. Mean aPWV, msSBP, msDBP, msPP, mean ambulatory (ma) BP, maPP and MAP will also be measured after 12 and 52 weeks of treatment.

Exploratory assessments comparing the two treatments after 12 and 52 weeks of treatment include pulse wave analysis (PWA) variables such as augmentation index (AIx), augmentation pressure, PP amplification ratio, duration of LV ejection and time to wave reflection; reduction in ma central (mac) $\mathrm{BP}$, macMAP and macPP; plasma biomarkers including NT-proBNP and urinary cyclic guanosine monophosphate/creatinine ratio and other biomarkers related to hypertension.

\section{Haemodynamic measurements}

The SphygmoCor X-CEL System (AtCor Medical, Sydney, Australia) is being used to non-invasively derive the ascending aortic pressure waveform from the brachial waveform using a validated generalised transfer function (GTF) ${ }^{23}$ A properly sized BP cuff is linked to a computer and software and the CASP, CAPP, augmentation pressure and AIx are determined from the analysis of the waveform by the system software.

The SphygmoCor X-CEL system also measures the carotid-femoral aPWV, the speed of the arterial pressure waveform as it travels through the descending aorta to the femoral artery, which is detected from simultaneously measured carotid and femoral arterial pulses. The carotid pulse is detected by applanation tonometry using a high-fidelity pressure transducer (Millar Instruments, Houston, Texas, USA), while the femoral pulse is detected using a partially inflated BP cuff

\section{Box 1 Inclusion criteria}

- Patients with essential hypertension aged $\geq 60$ years.

- Either untreated or treated with antihypertensive agents, and who have mean sitting systolic blood pressure (msSBP) $\geq 150$ and $<180 \mathrm{~mm} \mathrm{Hg}$ at randomisation are eligible to be included.

- Untreated patients (if they are newly diagnosed or have not been treated with antihypertensive drugs for 4 weeks prior to screening) must have msSBP $\geq 150$ and $<180 \mathrm{~mm} \mathrm{Hg}$ at screening and randomisation.

- Patients who have been treated with antihypertensive drugs during 4 weeks prior to screening must have msSBP $\geq 140$ and $<180 \mathrm{~mm} \mathrm{Hg}$ after 1 or 2 weeks of washout/placebo run-in and $\geq 150$ and $<180 \mathrm{~mm} \mathrm{Hg}$ at randomisation.

- Patients must have a difference in msSBP of within $\pm 15 \mathrm{~mm} \mathrm{Hg}$ between randomisation and the visit preceding randomisation.

- All patients must have a pulse pressure $>60 \mathrm{~mm} \mathrm{Hg}$ at randomisation.

- Patients who comply with all study requirements and demonstrate good medication compliance ( $\geq 80 \%$ compliance rate) during the single blind placebo run-in period. wrapped around the upper thigh. The distance travelled by the pulse wave is captured by making physical measurements on the body surface according to the manufacturer's recommendations. This new brachial cuff-based device with an individualised subdiastolic cuff pressure has recently been validated against the SphygmoCor device (AtCor Medical) using the classical radial tonometry-based methodology, and provides an operator-independent method to assess systolic pressure and aortic waveforms comparable with the existing validated tonometric-based methods. ${ }^{24}$ Measurements using the SphygmoCor X-CEL system will be performed at baseline, randomisation, week 12 or at the time of early discontinuation prior to week 12 , week 52 or at the time of early discontinuation between weeks 12 and 52 .

The $24 \mathrm{~h}$ maCAP and maPWA will be monitored using the oscillometric device, Mobil-O-Graph (IEM, Stolberg, Germany) with integrated ARC solver algorithms (Austrian Institute of Technology, Vienna, Austria). The traditional Mobil-O-Graph ambulatory BP monitoring device has been available for more than a decade and through several product generations. ${ }^{25-28}$ The actual BP measuring unit was validated according to the British Hypertension Society (BHS) and the European Society of Hypertension (ESH) recommendations. ${ }^{25} 27$ The method, equipped with a GTF to derive aortic pressure waveforms ${ }^{29}$ is based on brachial readings acquired in the course of the conventional pressure measurement at the diastolic level.

During the signal acquisition procedure, the received raw signals are separated into single waves and checked for their plausibility by means of extreme values and the corresponding wavelengths using a cross-correlation

\section{Box 2 Exclusion criteria}

- Malignant or severe hypertension or secondary causes of hypertension.

- History of atrial fibrillation during 3 months prior to screening or active atrial fibrillation or atrial flutter on ECG during 12 months prior to screening.

- History of cardiovascular disease (eg, myocardial infarction) during 12 months prior to screening.

- Existing angina pectoris requiring pharmacological therapy.

- Type 1 or 2 diabetes mellitus not well controlled based on the investigator's clinical judgement.

- Previous or current diagnosis of heart failure (NYHA Class II-IV), cardiac abnormalities such as second-degree or thirddegree atrioventricular block without a pacemaker, or malignancy.

- Evidence of severe renal impairment (eg, estimated glomerular filtration rate $<30 \mathrm{~mL} / \mathrm{min} / 1.73 \mathrm{~m}^{2}$ ).

- Laboratory abnormalities such as serum potassium $>5.5 \mathrm{mmol} / \mathrm{L}$.

- Known active liver disease or cirrhosis or evidence of hepatic disease.

- Patients requiring any drug treatment that could affect blood pressure.

Women of childbearing potential unless using highly effective methods of contraception during dosing. 
approach. Poor waveforms are removed from further processing. After applying the GTF to each waveform, the procedure is repeated. After final coherence verification, the quality judgement of grade ' 1 ' states that at least $80 \%$ of the waveforms were found to be eligible for further processing, while grades ' 2 ' and ' 3 ' represent a $\geq 50 \%$ and $<50 \%$ valid waveforms, respectively. ${ }^{30}$

Surrogates derived by this technique have been validated against solid-state catheter measurements and/ or compared with non-invasive readings (eg, tonometry and echocardiography) for aortic pressures, ${ }^{30-33}$ wave reflections ${ }^{34}$ or aPWV. ${ }^{35}{ }^{36}$ However, potential clinical usefulness has been demonstrated recently. ${ }^{37-39}$ Furthermore, feasibility $^{36}$ and reproducibility ${ }^{40}$ of cuff-based maPWA measurements have been reported. The Mobil-O-Graph maPWA monitor with integrated ARC solver algorithms holds approvals from CE, FDA and JPAL (among others).

\section{Safety assessments}

Safety and tolerability assessments include regular monitoring and recording of all adverse events and concomitant medications or significant non-drug therapies. Evaluations of routine blood chemistries, blood counts with white cell differential and urine analyses, physical examinations, ECGs and monitoring of vital signs will be performed at regular intervals.

\section{Statistical analysis plan}

A sample size of 183 completers per group is targeted, which is calculated based on the primary efficacy variable, change from baseline in mean CASP at 12 weeks, assuming an SD of $19 \mathrm{~mm} \mathrm{Hg}$. The sample size is calculated to ensure a power of $90 \%$ to detect statistical significance for the comparison of LCZ696-based treatment regimen with the olmesartan-based treatment regimen in assessing the superiority at the week 12 endpoint, under the alternative hypothesis that the treatment difference is $6.5 \mathrm{~mm} \mathrm{Hg}$ at a two-sided significance level of 0.05 . Assuming a $15 \%$ dropout rate, the total targeted sample size to be randomised is 432 patients (216 per group). The primary variable at the week 12 endpoint will be analysed using a two-way analysis of covariance (ANCOVA), with treatment and region as factors and the baseline as a covariate. Mean CAPP at the week 12 endpoint will be analysed using the same type of ANCOVA model used for the primary efficacy analysis.

\section{DISCUSSION}

Hypertension in patients over 60 years is often difficult to control because of age-associated adverse changes in vascular structure and function, especially arterial stiffening and the resulting changes in aortic haemodynamics. The majority of elderly patients present with features of arterial stiffening, notably isolated systolic hypertension (ISH), disrupted circadian BP variation, a non-dipping or early morning riser phenotype of hypertension and orthostatic hypertension. Moreover, compared with younger people, elderly patients are usually characterised with augmented aortic systolic and PP relative to brachial pressure, associated with diminished aortic-brachial pressure amplification resulting in the 'true' elevation of brachial systolic and PPs. In turn, the elevated aortic and brachial PPs that result from increased cardiac work to overcome the increased characteristic impedance of the aorta due to its age-related stiffening cause an increased predisposition to LV hypertrophy, myocardial ischaemia and heart failure.

Our hypothesis is that the ARNI LCZ696 provides a novel approach to neurohormonal modulation by concomitantly enhancing the NP system and suppressing the RAAS. Owing to the effects of enhanced NPs and RAAS inhibition, LCZ696 is anticipated to improve aortic stiffness, reduce characteristic impedance and improve central haemodynamics. NPs inhibit the production and action of vasoconstrictor peptides, inhibit sympathetic outflow and protect against excess salt and water retention. ${ }^{41}$ NPs also inhibit cardiac growth or the development of compensatory cardiac hypertrophy and regulate $\mathrm{CV}$ function. ${ }^{41}$ Inhibition of sympathetic tone might be beneficial in controlling morning surge in $\mathrm{BP}$ in elderly patients with hypertension. ${ }^{42} 43$ In addition, suppressing the RAAS offers the potential for many similar actions on CV structure and function as well as favourable effects on microcirculatory haemodynamics as evidenced by observations of reduced albuminuria and renal protection beyond what might have been anticipated from BP reduction alone.

It has already been demonstrated that LCZ696 provides superior reductions in msSBP and msDBP versus valsartan in an 8-week study including 1328 patients with mild-to-moderate hypertension. In addition, LCZ696 significantly reduced maSBP versus valsartan (betweentreatment difference: LCZ696 $200 \mathrm{mg}$ vs valsartan $160 \mathrm{mg},-3.23 \mathrm{~mm} \mathrm{Hg}, 95 \%$ CI -5.70 to -0.75 ; LCZ696 $400 \mathrm{mg}$ vs valsartan $320 \mathrm{mg},-5.14 \mathrm{~mm} \mathrm{Hg}, 95 \%$ CI -7.70 to -2.59$)$. However, there was no significant treatment difference in maDBP reductions, thereby providing evidence of improvements in PP with LCZ696 versus valsartan. These findings are consistent with improvements in large artery function. Furthermore, BP control rates were significantly higher with LCZ696 $200 \mathrm{mg}$ than with $160 \mathrm{mg}$ valsartan $(46 \% \quad(78 / 168)$ vs $33 \% \quad(54 / 163)$, $\mathrm{p}=0.0147)$. Importantly, in these studies, unlike the vasopeptidase inhibitor omapatrilat, LCZ696 was generally well tolerated without an incidence of angio-oedema during 8 weeks of treatment. ${ }^{21}$

The reported improvements in PP suggest the potential for LCZ696 to protect more effectively than the existing BP-lowering agents from several consequences of ISH and vascular stiffness, such as stroke and diastolic heart failure. However, in the study by Ruilope et $a l^{21}$ CAP, central haemodynamics and arterial stiffness were not assessed, precluding a meticulous assessment of the mechanisms underpinning the superior antihypertensive properties of LCZ696. 
In the PARAMETER study, the measurement of CAP should more accurately assess the loading conditions on the LV, myocardium, coronary arteries and cerebral vasculature, and therefore, theoretically, CAP should provide a basis for more effective protection against $\mathrm{CV}$ target organ damage and events compared with brachial pressures. In this regard, even in normotensive individuals, measurement of aortic BP enhances the ability to predict the target organ changes. ${ }^{44}$

The PARAMETER study was initiated in December 2012, with a novel design to evaluate central haemodynamics in elderly patients with ISH and a widened PP at 12 and 52 weeks of treatment with LCZ696 or ARB olmesartan. If the changes in CASP at 12 weeks (acute haemodynamic effect) are larger than that in SBP, this would support the hypothesis that LCZ696 has the potential to favourably impact aortic haemodynamics and improve ventricular-vascular coupling in elderly patients with aged aortas and ISH. Treatment differences in PWV at 52 weeks would further support a direct beneficial effect of LCZ696 on aortic stiffness due to structural changes, independent of MAP, as both treatment groups will be titrated to achieve similar BP control. The overall effect of LCZ696 on maBP (maSBP, maDBP and maPP), $24 \mathrm{~h}$ BP variability (SD, covariance) and circadian BP rhythms (nocturnal dipping status and morning surge) will also be assessed in comparison with olmesartan. The study targets randomisation of 432 patients and final results are expected in 2015. We acknowledge the limitations of this study which is designed to look at surrogate markers rather than major CV outcomes; nevertheless, this is important to establish whether there are differential effects of drugs therapies on surrogate outcomes of CV disease to justify and impact on the design of subsequent phase III studies assessing the potential of LCZ696 for enhanced CV disease and stroke prevention in elderly patients with ISH.

In addition to the PARAMETER study, the efficacy and safety of LCZ696 are being evaluated in related studies, for example, in comparison with olmesartan in elderly patients with mild-to-moderate hypertension, in patients with systolic hypertension and in patients with systolic hypertension who did not respond to olmesartan. Although it is hypothesised in the PARAMETER study that LCZ696 will be more effective at lowering central aortic and brachial BP compared with olmesartan, it is also recognised that other agents may need to be added to reach recommended BP goals in the elderly patients with systolic hypertension. Calcium channel blockers (CCBs, usually amlodipine) and diuretics are the most commonly used antihypertensive agents in combination with RAAS blockade for patients failing to achieve their BP goal with RAAS blockade monotherapy. Besides being very effective, such combination therapies of specific antihypertensive classes may also improve safety and tolerability. For example, addition of ARBs to CCBs has been shown to reduce the peripheral oedema associated with amlodipine monotherapy. ${ }^{45}$ Similarly, diureticinduced hypokalaemia has been shown to be attenuated when RAAS blockade is combined with diuretic therapy. ${ }^{46}$ To further evaluate such combination therapies with LCZ696, the BP-lowering efficacy, safety and tolerability are being evaluated in combination with amlodipine in patients with mild-to-moderate hypertension, who were non-responsive to amlodipine, and in patients with systolic hypertension. There are also other trials investigating the efficacy and safety of LCZ696 in patients with severe hypertension and in patients with renal impairment.

In summary, the PARAMETER study will evaluate mechanisms associated with BP lowering in elderly patients with an aged CV system as evidenced by systolic hypertension and a widened PP. The study will define whether LCZ696, a first-in-class ARNI, is more effective in lowering CASP and CAPP than ARB olmesartan and also explore whether this effect is related to a BP-independent reduction in arterial stiffening, suggesting a novel mechanism to target systolic hypertension, a major and increasingly important unmet therapeutic need in the management of hypertension in elderly patients.

\section{Author affiliations}

${ }^{1}$ Department of Cardiovascular Sciences and National Institute for Health Research (NIHR) University College London (UCL) Hospitals Biomedical Research Centre, University College London, London, UK

${ }^{2}$ Department of Cardiology, University of Cardiff, Wales, UK

${ }^{3}$ Jichi Medical School, Tochigi, Japan

${ }^{4}$ Novartis Pharmaceuticals Corporation, East Hanover, New Jersey, USA

${ }^{5}$ Novartis Pharma AG, Basel, Switzerland

Acknowledgements The authors acknowledge Dr Sreedevi Boggarapu (Novartis Healthcare Pvt. Ltd, Hyderabad, India) for medical writing and editorial support. They also thank all the clinical investigators and study coordinators at the participating centres and all the patients who participate in the study.

Contributors All authors have contributed to the design of this study and have contributed to the drafting of the manuscript.

Funding This study is funded by Novartis Pharma AG, Basel, Switzerland. BW is a NIHR Senior Investigator and is supported by the NIHR UCL Hospitals Biomedical Research Centre.

Competing interests BW, JRC and KK have received honoraria from Novartis for consulting and presentations at scientific symposia. DHZ, PC, AH, PB and $\mathrm{JZ}$ are employees of Novartis and are thus eligible for Novartis stock and stock options.

Ethics approval This study has been approved by all relevant ethics committees- 51 sites.

Provenance and peer review Not commissioned; externally peer reviewed.

Data sharing statement No additional data are available.

Clinical trials identifier: EUDract number 2012-002899-14 and ClinicalTrials.gov NCT01692301.

Open Access This is an Open Access article distributed in accordance with the Creative Commons Attribution Non Commercial (CC BY-NC 3.0) license, which permits others to distribute, remix, adapt, build upon this work noncommercially, and license their derivative works on different terms, provided the original work is properly cited and the use is non-commercial. See: http:// creativecommons.org/licenses/by-nc/3.0/ 


\section{REFERENCES}

1. Kearney PM, Whelton M, Reynolds K, et al. Global burden of hypertension: analysis of worldwide data. Lancet 2005;365:217-23.

2. Lawes CM, Vander HS, Rodgers A. Global burden of blood-pressure-related disease, 2001. Lancet 2008;371:1513-18.

3. Lionakis $\mathrm{N}$, Mendrinos $\mathrm{D}$, Sanidas $\mathrm{E}$, et al. Hypertension in the elderly. World J Cardiol 2012;4:135-47.

4. Arguedas JA, Perez MI, Wright JM. Treatment blood pressure targets for hypertension. Cochrane Database Syst Rev 2009;(3): CD004349.

5. Franklin SS, Jacobs MJ, Wong ND, et al. Predominance of isolated systolic hypertension among middle-aged and elderly US hypertensives: analysis based on National Health and Nutrition Examination Survey (NHANES) III. Hypertension 2001;37:869-74.

6. Vasan RS, Beiser A, Seshadri S, et al. Residual lifetime risk for developing hypertension in middle-aged women and men: the Framingham Heart Study. JAMA 2002;287:1003-10.

7. Palatini P, Casiglia E, Gasowski J, et al. Arterial stiffness, central hemodynamics, and cardiovascular risk in hypertension. Vasc Health Risk Manag 2011;7:725-39.

8. Vlachopoulos C, Aznaouridis K, O'Rourke MF, et al. Prediction of cardiovascular events and all-cause mortality with central haemodynamics: a systematic review and meta-analysis. Eur Heart J 2010;31:1865-71.

9. Mitchell GF, Vasan RS, Keyes MJ, et al. Pulse pressure and risk of new-onset atrial fibrillation. JAMA 2007;297:709-15.

10. Waldstein SR, Rice SC, Thayer JF, et al. Pulse pressure and pulse wave velocity are related to cognitive decline in the Baltimore Longitudinal Study of Aging. Hypertension 2008;51:99-104.

11. Ford ML, Tomlinson LA, Chapman TP, et al. Aortic stiffness is independently associated with rate of renal function decline in chronic kidney disease stages 3 and 4. Hypertension 2010;55:1110-15.

12. Manisty $\mathrm{CH}$, Hughes AD. Meta-analysis of the comparative effects of different classes of antihypertensive agents on brachial and central systolic blood pressure, and augmentation index. Br J Clin Pharmacol 2013;75:79-92

13. Roman MJ, Devereux RB, Kizer JR, et al. Central pressure more strongly relates to vascular disease and outcome than does brachial pressure: the Strong Heart Study. Hypertension 2007;50:197-203.

14. Roman MJ, Okin PM, Kizer JR, et al. Relations of central and brachial blood pressure to left ventricular hypertrophy and geometry: the Strong Heart Study. J Hypertens 2010;28:384-8.

15. Wang KL, Cheng HM, Chuang SY, et al. Central or peripheral systolic or pulse pressure: which best relates to target organs and future mortality? J Hypertens 2009;27:461-7.

16. Jankowski P, Kawecka-Jaszcz K, Czarnecka D, et al. Ascending aortic, but not brachial blood pressure-derived indices are related to coronary atherosclerosis. Atherosclerosis 2004;176:151-5.

17. Ostergren J, Poulter NR, Sever PS, et al. The Anglo-Scandinavian Cardiac Outcomes Trial: blood pressure-lowering limb: effects in patients with type II diabetes. J Hypertens 2008;26:2103-11.

18. Williams B, Lacy PS, Thom SM, et al. Differential impact of blood pressure-lowering drugs on central aortic pressure and clinical outcomes: principal results of the Conduit Artery Function Evaluation (CAFE) study. Circulation 2006;113:1213-25.

19. Mitchell GF, Izzo JL Jr, Lacourciere Y, et al. Omapatrilat reduces pulse pressure and proximal aortic stiffness in patients with systolic hypertension: results of the conduit hemodynamics of omapatrilat international research study. Circulation 2002;105:2955-61.

20. Veelken R, Schmieder RE. Neutral endopeptidase inhibition: the potential of a new therapeutic approach in cardiovascular disease evolves. J Hypertens 2002;20:599-603.

21. Ruilope LM, Dukat A, Bohm M et al. Blood-pressure reduction with LCZ696, a novel dual-acting inhibitor of the angiotensin II receptor and neprilysin: a randomised, double-blind, placebo-controlled, active comparator study. Lancet 2010;375:1255-66.

22. Solomon SD, Zile M, Pieske B, et al. The angiotensin receptor neprilysin inhibitor LCZ696 in heart failure with preserved ejection fraction: a phase 2 double-blind randomised controlled trial. Lancet 2012;380:1387-95.

23. Gallagher D, Adji A, O'Rourke MF. Validation of the transfer function technique for generating central from peripheral upper limb pressure waveform. Am J Hypertens 2004;17:1059-67.
24. Butlin M, Qasem A, Avolio AP. Estimation of central aortic pressure waveform features derived from the brachial cuff volume displacement waveform. Conf Proc IEEE Eng Med Biol Soc 2012;2012:2591-4.

25. Franssen PM, Imholz BP. Evaluation of the Mobil-O-Graph new generation ABPM device using the ESH criteria. Blood Press Monit 2010;15:229-31.

26. Jones $\mathrm{CR}$, Taylor $\mathrm{K}$, Chowienczyk $\mathrm{P}$, et al. A validation of the Mobil O Graph (version 12) ambulatory blood pressure monitor. Blood Press Monit 2000;5:233-8.

27. Wei W, Tolle M, Zidek W, et al. Validation of the mobil-O-Graph: 24 h-blood pressure measurement device. Blood Press Monit 2010;15:225-8

28. Westhoff $\mathrm{TH}$, Straub-Hohenbleicher $\mathrm{H}$, Schmidt $\mathrm{S}$, et al. Convenience of ambulatory blood pressure monitoring: comparison of different devices. Blood Press Monit 2005;10:239-42.

29. Wassertheurer S, Mayer C, Breitenecker F. Modeling arterial and left ventricular coupling for non-invasive measurements. Simulation Model Pract Theory 2008;16:988-97.

30. Wassertheurer S, Kropf J, Weber T, et al. A new oscillometric method for pulse wave analysis: comparison with a common tonometric method. J Hum Hypertens 2010;24:498-504.

31. Nunan D, Wassertheurer S, Lasserson D, et al. Assessment of central haemomodynamics from a brachial cuff in a community setting. BMC Cardiovasc Disord 2012;12:48.

32. Weber T, Wassertheurer S, Rammer M, et al Validation of a brachial cuff-based method for estimating central systolic blood pressure. Hypertension 2011;58:825-32.

33. Weiss W, Gohlisch C, Harsch-Gladisch C, et al. Oscillometric estimation of central blood pressure: validation of the Mobil-O-Graph in comparison with the SphygmoCor device. Blood Press Monit 2012;17:128-31.

34. Hametner B, Wassertheurer S, Kropf J, et al. Wave reflection quantification based on pressure waveforms alone-methods, comparison, and clinical covariates. Comput Methods Programs Biomed 2013;109:250-9.

35. Hametner B, Wassertheurer S, Kropf J, et al. Oscillometric estimation of aortic pulse wave velocity: comparison with intra-aortic catheter measurements. Blood Press Monit 2013;18:173-6.

36. Luzardo L, Lujambio I, Sottolano M, et al. 24-h ambulatory recording of aortic pulse wave velocity and central systolic augmentation: a feasibility study. Hypertens Res 2012;35:980-7.

37. Reppel M, Franzen K, Bode F, et al. Central hemodynamics and arterial stiffness during the finals of the world cup soccer championship 2010. Int J Cardiol 2013;166:627-32.

38. Weber T, Wassertheurer S, Rammer M, et al. Wave reflections, assessed with a novel method for pulse wave separation, are associated with end-organ damage and clinical outcomes. Hypertension 2012;60:534-41.

39. Weber T, Wassertheurer S, O'Rourke MF, et al. Pulsatile hemodynamics in patients with exertional dyspnea: potentially of value in the diagnostic evaluation of suspected heart failure with preserved ejection fraction. J Am Coll Cardiol 2013;61:1874-83.

40. Protogerou AD, Argyris A, Nasothimiou E, et al. Feasibility and reproducibility of noninvasive 24-h ambulatory aortic blood pressure monitoring with a brachial cuff-based oscillometric device. Am J Hypertens 2012;25:876-82.

41. Levin ER, Gardner DG, Samson WK. Natriuretic peptides. N Engl J Med 1998:339:321-8.

42. London GM, Weiss YA, Pannier BP, et al. Tilt test in essential hypertension. Differential responses in heart rate and vascular resistance. Hypertension 1987;10:29-34.

43. Okada Y, Galbreath MM, Shibata S, et al. Morning blood pressure surge is associated with arterial stiffness and sympathetic baroreflex sensitivity in hypertensive seniors. Am J Physiol Heart Circ Physiol 2013;305:H793-802.

44. Booysen HL, Norton GR, Maseko MJ, et al. Aortic, but not brachial blood pressure category enhances the ability to identify target organ changes in normotensives. J Hypertens 2013;31:1124-30.

45. Chazova IE, Dongre N, Vigdorchik AV. Real-life safety and effectiveness of amlodipine/valsartan combination in the treatment of hypertension. Adv Ther 2011:28:134-49.

46. Palmer BF. Improving BP control with combined renin-angiotensin system blockade and thiazide diuretics in hypertensive patients with diabetes mellitus or kidney disease. Am J Cardiovasc Drugs 2008;8:9-14. 